\title{
YQTLs improve Qi-deficiency and blood stasis in multiple cerebral infarction rats by increasing the expression of VEGFR2 and CD31
}

\author{
Bing Jie Xue ${ }^{1}$, Ji Sheng Huang ${ }^{2}$, Bo Ma ${ }^{1}$, Ting Ting Hao ${ }^{1}$, Jian Xun Liu ${ }^{1}$ \\ ${ }^{1}$ Institute of Basic Medical Sciences, Xiyuan Hospital, China Academy of Chinese Medical Sciences, China, ${ }^{2}$ Research \\ Institute of Shineway Pharmaceutical Group, Hebei 065201, China
}

This research was aimed to evaluate the protective effect of Yi Qi Tong Luo particles (YQTLs), and explore the potential mechanism associated to angiogenesis using proteomic technology and immunohistochemical. Methods: Firstly, we established an animal model of multiple cerebral infarction (MCI) with qi deficiency and blood stasis. Rats were randomly divided into six groups: SHAM group, Vehicle group, Bu Yang Huan Wu Original decoction group (BYHWO), EGb761 group, high and low dose of YQTLs group. Rats were undergoing sleep deprivation after one week of MCI and detected the tongues and pulses of rats after six months of sleep deprivation, followed by collecting blood to analysis the four coagulation indicator. Brain tissue was taken out to measure the area of cerebral infarction and differential expression of angiogenesis associated proteins using proteomic research and verify the angiogenesis marker protein VEGFR2 and CD31 by immunohistochemical. Results: $G$ and $B$ value was decreased significantly and the pulse distension, the activated partial thromboplatin time (APTT) as well as prothrombin time (PT) were recovered following YQTLs treatment. The results of proteomic technology showed that proteins associated with angiogenesis are reversed compared with Vehicle group. Moreover, the expression of VEGFR2 and CD31 from immunohistochemical was de-depressed after YQTLs treatment. Conclusion: the MCI with qi deficiency and blood stasis was alleviated obviously following YQTLs treatment and the possible mechanism is that the YQTLs may promote angiogenesis during cerebral ischemia. 